\title{
GOLD PRICESFORECASTING USING TRIPLE EXPONENTIAL METHOD
}

\author{
Khairawati $^{1}$, Wahyu Fuadi ${ }^{2}$, Rizki Ramadhansyah ${ }^{3}$, Dedi Fariadi ${ }^{4}$ \\ ${ }^{1}$ D-III Secretariat Study Program, Malikussaleh University \\ ${ }^{2}$ Informatics Engineering Study Program, Malikussaleh University \\ ${ }^{3,4}$ Department of Electrical Engineering, Malikussaleh University \\ Email: khairawati@unimal.ac.id, wahyu.fuadi@unimal.ac.id, dedi.fariadi@unimal.ac.id \\ *khairawati@unimal.ac.id
}

\begin{abstract}
Governments, organizations, and citizens have taken an interest in gold price fluctuations. Gold price forecasting that is accurate may effectively capture price shift tendencies and reduce the effects of gold market volatility. However, due to the multi-factor and nonlinear nature of the gold market. The triple exponential smoothing strategy is used in this study to predict the rise in a value over time since it can replicate trends and seasonal patterns. according to the gold price swings pattern and seasonal components at the same time To calculate system accuracy, the Mean Absolute Percentage Error is employed (MAPE). With alpha 0.15 and beta 0.85 as parameter values, the triple exponential smoothing (TES) approach achieves an accuracy rate of 86.93 percent and a MAPE of 12.49 percent in this study.
\end{abstract}

Keyword :gold price,market volatility,gold market,trends,seasonal patterns

\section{INTRODUCTION}

Gold price forecasting is a crucial financial problem since governments, scholars, and investors all need to be informed of shifting patterns. Gold is a one-of-a-kind precious metal that combines the characteristics of commodities, valuable metals, and money(FAUSTINA et al., 2017).Gold is a necessary raw commodity for both industrial production and the aesthetics of jewelry. As a result, the gold market is receiving a lot of attention from the domestic and international population, institutional investors, and governments(Istamar et al., 2019).The gold market's importance is growing in tandem with the financial markets, and it is quickly becoming a financial investment market as significant as the stock market, futures market, bond market, and so on(Evamelia\& Panjaitan, 2019).The gold market is a global market with a nonlinear system that allows for fast price and volume fluctuations(Chen et al., 2019).In response to the essential and hard task of anticipating gold price changes, which is being undertaken by both researchers and the government(Zhang \& $\mathrm{Ci}, 2021)$.It is crucial to anticipate changes precisely and effectively in the price of gold, both theoretically and practically(ALTAN \& KARASU, 2019).

Forecasting gold price variations has investigated several gold price prediction techniques, ranging from statistical models to moving average models(Qasim et al., 2021).Currently, the most often used statistical model is autoregressive conditional heteroscedasticity $(\mathrm{ARCH})($ Ayele et al., 2021).In recent years, gold is a precious metal that is in great demand, especially Antam Gold or 24-carat Precious Metal, both for investment and as jewelry(Prananingtyas, 2018).This is indicated by data on gold jewelry demand in Indonesia in the fourth quarter of 2015, which increased by 16.88 percent year over year from 7.7 to 9 tons(Juhro\& Iyke, 2021). The total amount of demand in 2015 was 38.9 tons. According to data from the World Gold Council (WGC), Indonesia became the Southeast Asia country with the greatest level of demand in the fourth quarter(Humphreys, 2017). 
Prediction is the technique of estimating (measuring) the amount or amount of something in the future based on prior data (time series) that has been properly studied, particularly using statistical methods(Majid, 2018).Gold price prediction aims to determine future investment possibilities for gold prices so that gold investors may use it to determine changes in gold prices(Alameer et al., 2019).

The technique of anticipating future occurrences is known as forecasting. Forecasting is used to decrease uncertainty and offer standards for evaluating actual performance(de Oliveira \& Cyrino Oliveira, 2018). Forecasting can help customers forecast the price of all sorts of things that have a sale and buy value. The triple exponential approach is used in this study to forecast gold prices and assess the pattern of growing gold prices using data obtained from national and regional sources.

\section{LITERATURE REVIEW \\ Exponential Smoothing (ES)}

Exponential Smoothing is a method that continuously computes utilizing the most recent data based on finding the average exponential smoothing of the preceding data(de Oliveira \& Cyrino Oliveira, 2018). This method puts data to the test by giving weight to observations to forecast the future. The most recent observation period on ES is more predictive than the prior observation period(Barrow et al., 2021). The parameters (alpha), (beta), and are used to calculate this weighting value (gamma). SES, DES, and TES are all distinct approaches. Single Exponential Smoothing is a variant of the moving average technique, which involves assigning a value to data in a time series. This method works best with data variations that may be classified as steady, meaning that there is no pattern or regular movement in the ups and downs. This method is used to generate predictions(Majid, 2018).

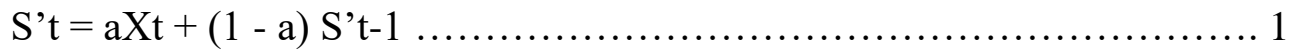

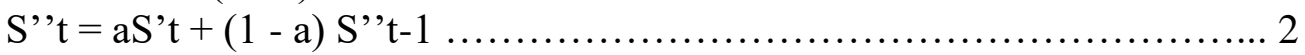

Triple Exponential Smoothing is an approach for dealing with seasonal impacts and trend features that emerge on time series data at the same time. This method necessitates the inclusion of a new variable, frequency, to denote the number of periods in each cycle. The multiplicative seasonal model and the additive seasonal model are two models that might be utilized in this method depending on the season. The multiplicative model's key feature is that the amplitude of seasonal variations fluctuates and is dependent on the smoothing of the entire time series. This approach is useful when there is a trend or a seasonal pattern that increases with increasing data amount.

\section{Triple Exponential Smoothing Method}

The Triple Exponential Smoothing technique is used to forecast the price of gold for a single day. This technique will process today's gold price data with method parameters that may be generated randomly or via trial and error to estimate the gold price for the next day. Ten gold price data points were collected sequentially from the preceding several months to assess the capability of this procedure, which was measured by the level of accuracy.

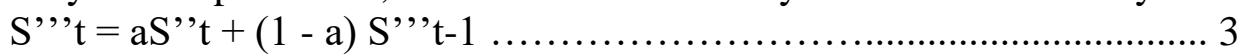

\section{RESULTS AND DISCUSSION}

The Triple Exponential Smoothing technique is employed in this study to forecast the price of gold over a one-day period. This approach will process today's gold price data by employing method parameters selected at random or by trial and error to estimate gold prices 


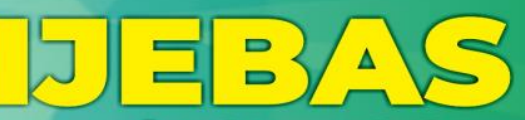

Themational Journal of Economic, Business,

Ace्unting, Acriculture Management and Sharia Administration

for tomorrow. Data on gold prices can be taken up to 10 times in a row. The strategy will then be tested using gold price data from the preceding several months to determine its ability as evaluated by the amount of accuracy of its success. Then proceed to perform manual computations. In this manual computation, gold price data from 01 August 2021 to 26 September 2021 is used as a test of this approach, with 5 sources of gold prices for each date.

Table 1

Gold price data

\begin{tabular}{|c|c|c|c|c|c|}
\hline \multirow{2}{*}{ Date } & \multicolumn{5}{|c|}{ Gold Price Data Source (0.7 grams) } \\
\hline & 1 & 2 & 3 & 4 & 5 \\
\hline $01 / 08 / 2021$ & $\begin{array}{r}\mathrm{Rp} \\
693.520,79\end{array}$ & $\begin{array}{r}\mathrm{Rp} \\
693.826,15\end{array}$ & $\begin{array}{r}\mathrm{Rp} \\
697.867,08\end{array}$ & $\begin{array}{r}\mathrm{Rp} \\
653,105.00\end{array}$ & $\begin{array}{r}\mathrm{Rp} \\
652,901.00\end{array}$ \\
\hline 02/08/2021 & $\begin{array}{r}\mathrm{Rp} \\
622.859,18\end{array}$ & $\begin{array}{r}\mathrm{Rp} \\
698.781,23\end{array}$ & $\begin{array}{r}\mathrm{Rp} \\
697.275,34\end{array}$ & $\begin{array}{r}\mathrm{Rp} \\
692,338.00\end{array}$ & $\begin{array}{r}\mathrm{Rp} \\
732,686.00\end{array}$ \\
\hline 03/08/2021 & $\begin{array}{r}\mathrm{Rp} \\
704.608,96\end{array}$ & $\begin{array}{r}\mathrm{Rp} \\
705.225,78\end{array}$ & $\begin{array}{r}\mathrm{Rp} \\
695.475,24\end{array}$ & $\begin{array}{r}\mathrm{Rp} \\
642,972.00\end{array}$ & $\begin{array}{r}\mathrm{Rp} \\
687,117.00\end{array}$ \\
\hline 04/08/2021 & $\begin{array}{r}\mathrm{Rp} \\
702.615,63\end{array}$ & $\begin{array}{r}\mathrm{Rp} \\
705.299,84\end{array}$ & $\begin{array}{r}\mathrm{Rp} \\
708.033,59\end{array}$ & $\begin{array}{r}\mathrm{Rp} \\
682,412.00\end{array}$ & $\begin{array}{r}\mathrm{Rp} \\
734,906.00\end{array}$ \\
\hline 05/08/2021 & $\begin{array}{r}\mathrm{Rp} \\
703.852,69\end{array}$ & $\begin{array}{r}\mathrm{Rp} \\
688.784,92\end{array}$ & $\begin{array}{r}\mathrm{Rp} \\
708.538,27\end{array}$ & $\begin{array}{r}\mathrm{Rp} \\
696,599.00\end{array}$ & $\begin{array}{r}\mathrm{Rp} \\
663,294.00\end{array}$ \\
\hline $22 / 08 / 2021$ & $\begin{array}{r}\mathrm{Rp} \\
687.817,27\end{array}$ & $\begin{array}{r}\mathrm{Rp} \\
701.089,88\end{array}$ & $\begin{array}{r}\mathrm{Rp} \\
685.324,00\end{array}$ & $\begin{array}{r}\mathrm{Rp} \\
685,736.00\end{array}$ & $\begin{array}{r}\mathrm{Rp} \\
674,889.00\end{array}$ \\
\hline 23/08/2021 & $\begin{array}{r}\mathrm{Rp} \\
689.074,29\end{array}$ & $\begin{array}{r}\mathrm{Rp} \\
689.503,11\end{array}$ & $\begin{array}{r}\mathrm{Rp} \\
686.710,86\end{array}$ & $\begin{array}{r}\mathrm{Rp} \\
709,371.00\end{array}$ & $\begin{array}{r}\mathrm{Rp} \\
658,334.00\end{array}$ \\
\hline $24 / 08 / 2021$ & $\begin{array}{r}\mathrm{Rp} \\
696.947,92\end{array}$ & $\begin{array}{r}\mathrm{Rp} \\
695.954,72\end{array}$ & $\begin{array}{r}\mathrm{Rp} \\
690.243,62\end{array}$ & $\begin{array}{r}\mathrm{Rp} \\
696,004.00\end{array}$ & $\begin{array}{r}\mathrm{Rp} \\
727,635.00\end{array}$ \\
\hline $25 / 08 / 2021$ & $\begin{array}{r}\mathrm{Rp} \\
685.426,79\end{array}$ & $\begin{array}{r}\mathrm{Rp} \\
685.257,26\end{array}$ & $\begin{array}{r}\mathrm{Rp} \\
696.552,12\end{array}$ & $\begin{array}{r}\mathrm{Rp} \\
646,640.00\end{array}$ & $\begin{array}{r}\mathrm{Rp} \\
702,968.00\end{array}$ \\
\hline 26/08/2021 & $\begin{array}{r}\mathrm{Rp} \\
687.434,13\end{array}$ & $\begin{array}{r}\mathrm{Rp} \\
687.671,65\end{array}$ & $\begin{array}{r}\mathrm{Rp} \\
683.430,44\end{array}$ & $\begin{array}{r}\mathrm{Rp} \\
669,523.00\end{array}$ & $\begin{array}{r}\mathrm{Rp} \\
652,083.00\end{array}$ \\
\hline $01 / 09 / 2021$ & $\begin{array}{r}\mathrm{Rp} \\
685.610,96\end{array}$ & $\begin{array}{r}\mathrm{Rp} \\
686.227,56\end{array}$ & $\begin{array}{r}\mathrm{Rp} \\
676.364,92\end{array}$ & $\begin{array}{r}\mathrm{Rp} \\
696,749.00\end{array}$ & $\begin{array}{r}\mathrm{Rp} \\
725,764.00\end{array}$ \\
\hline $02 / 09 / 2021$ & $\begin{array}{r}\mathrm{Rp} \\
686.586,44\end{array}$ & $\begin{array}{r}\mathrm{Rp} \\
686.056,88\end{array}$ & $\begin{array}{r}\mathrm{Rp} \\
684.944,48\end{array}$ & $\begin{array}{r}\mathrm{Rp} \\
734,301.00\end{array}$ & $\begin{array}{r}\mathrm{Rp} \\
678,965.00\end{array}$ \\
\hline 03/09/2021 & $\begin{array}{r}\mathrm{Rp} \\
685.598,10\end{array}$ & $\begin{array}{r}\mathrm{Rp} \\
684.908,58\end{array}$ & $\begin{array}{r}\mathrm{Rp} \\
689.234,26\end{array}$ & $\begin{array}{r}\mathrm{Rp} \\
713,427.00\end{array}$ & $\begin{array}{r}\mathrm{Rp} \\
670,410.00\end{array}$ \\
\hline 04/09/2021 & $\begin{array}{r}\mathrm{Rp} \\
683.677,41\end{array}$ & $\begin{array}{r}\mathrm{Rp} \\
682.718,40\end{array}$ & $\begin{array}{r}\mathrm{Rp} \\
684.944,48\end{array}$ & $\begin{array}{r}\mathrm{Rp} \\
656,311.00\end{array}$ & $\begin{array}{r}\mathrm{Rp} \\
675,300.00\end{array}$ \\
\hline
\end{tabular}


Gold Pricesforecasting Using Triple Exponential Method

DOI: $10.54443 /$ ijebas.v1i2.79

\begin{tabular}{|c|c|c|c|c|c|}
\hline $05 / 09 / 2021$ & $\begin{array}{r}\mathrm{Rp} \\
683.677,41\end{array}$ & $\begin{array}{r}\mathrm{Rp} \\
687.890,65\end{array}$ & $\begin{array}{r}\mathrm{Rp} \\
683.935,12\end{array}$ & $\begin{array}{r}\mathrm{Rp} \\
712,308.00\end{array}$ & $\begin{array}{r}\mathrm{Rp} \\
671,319.00\end{array}$ \\
\hline $22 / 09 / 2021$ & $\begin{array}{r}\mathrm{Rp} \\
671.597,84\end{array}$ & $\begin{array}{r}\mathrm{Rp} \\
671.966,53\end{array}$ & $\begin{array}{r}\mathrm{Rp} \\
674.093,86\end{array}$ & $\begin{array}{r}\mathrm{Rp} \\
675,309.00\end{array}$ & $\begin{array}{r}\mathrm{Rp} \\
724,898.00\end{array}$ \\
\hline $23 / 09 / 2021$ & $\begin{array}{r}\mathrm{Rp} \\
677.855,40\end{array}$ & $\begin{array}{r}\mathrm{Rp} \\
678.565,19\end{array}$ & $\begin{array}{r}\mathrm{Rp} \\
671.822,80\end{array}$ & $\begin{array}{r}\mathrm{Rp} \\
653,508.00\end{array}$ & $\begin{array}{r}\mathrm{Rp} \\
691,057.00\end{array}$ \\
\hline $24 / 09 / 2021$ & $\begin{array}{r}\mathrm{Rp} \\
677.816,53\end{array}$ & $\begin{array}{r}\mathrm{Rp} \\
677.639,10\end{array}$ & $\begin{array}{r}\mathrm{Rp} \\
683.935,12\end{array}$ & $\begin{array}{r}\mathrm{Rp} \\
682,275.00\end{array}$ & $\begin{array}{r}\mathrm{Rp} \\
714,812.00\end{array}$ \\
\hline $25 / 09 / 2021$ & $\begin{array}{r}\mathrm{Rp} \\
678.848,05\end{array}$ & $\begin{array}{r}\mathrm{Rp} \\
674.584,65\end{array}$ & $\begin{array}{r}\mathrm{Rp} \\
678.635,98\end{array}$ & $\begin{array}{r}\mathrm{Rp} \\
700,371.00\end{array}$ & $\begin{array}{r}\mathrm{Rp} \\
669,084.00\end{array}$ \\
\hline 26/09/2021 & $\begin{array}{r}\mathrm{Rp} \\
678.848,05\end{array}$ & $\begin{array}{r}\mathrm{Rp} \\
673.098,00\end{array}$ & $\begin{array}{r}\mathrm{Rp} \\
677.987,34\end{array}$ & $\begin{array}{r}\mathrm{Rp} \\
724,239.00\end{array}$ & $\begin{array}{r}\mathrm{Rp} \\
670,835.00\end{array}$ \\
\hline
\end{tabular}

\section{MAPE (Mean Absolute Percentage Error)}

The mean absolute percentage error is calculated by dividing the absolute error in each period by the actual value observed at that moment (MAPE). After that, the absolute percentage errors are averaged. When the size or magnitude of the forecasting variable is relevant in determining prediction accuracy, this strategy is useful. The size of the anticipated error in proportion to the real value is measured with MAPE.

$$
\begin{aligned}
\text { MAPE }=\frac{\mathbf{1 0 0} \%}{\mathbf{1}} \sum_{t=1}^{n} \frac{|\boldsymbol{A t}-\boldsymbol{F} \boldsymbol{t}|}{\boldsymbol{A} \boldsymbol{t}} \\
=\frac{100 \%}{1} \sum_{t=1}^{n} \frac{\mid \text { Rp. } 693.520 .79-\mathrm{Rp} 574,665.384 \mid}{\text { Rp. } 693.520 .79}=17,14 \%
\end{aligned}
$$

Table. 2

Mean Absolute Percentage Error (MAPE)

\begin{tabular}{|c|r|r|c|}
\hline Date & $\begin{array}{c}\text { Actual } \\
\text { Price 1 }\end{array}$ & Forecast 5 & MAPE \\
\hline $01 / 08 / 2$ & $\mathrm{Rp}$ & $\mathrm{Rp}$ & 17.14 \\
021 & $693.520,79$ & $574,665.384$ & $\%$ \\
\hline $02 / 08 / 2$ & $\mathrm{Rp}$ & $\mathrm{Rp}$ & \\
021 & $622.859,18$ & $632,332.392$ & $1.52 \%$ \\
\hline $03 / 08 / 2$ & $\mathrm{Rp}$ & $\mathrm{Rp}$ & 16.98 \\
021 & $704.608,96$ & $584,994.756$ & $\%$ \\
\hline $04 / 08 / 2$ & $\mathrm{Rp}$ & $\mathrm{Rp}$ & 12.27 \\
021 & $702.615,63$ & $616,428.656$ & $\%$ \\
\hline
\end{tabular}


Thremational Journal of Economic, Business,

Accounting, Agriculture Management and Sharia Administration

\begin{tabular}{|c|c|c|c|}
\hline $\begin{array}{l}05 / 08 / 2 \\
021\end{array}$ & $\begin{array}{r}\mathrm{Rp} \\
703.852,69\end{array}$ & $\begin{array}{c}\mathrm{Rp} \\
590,783.876\end{array}$ & $\begin{array}{l}16.06 \\
\%\end{array}$ \\
\hline $\begin{array}{l}\text { 22/08/2 } \\
021\end{array}$ & $\begin{array}{r}\mathrm{Rp} \\
687.817,27\end{array}$ & $\begin{array}{c}\mathrm{Rp} \\
590,962.084\end{array}$ & $\begin{array}{l}14.08 \\
\%\end{array}$ \\
\hline $\begin{array}{l}23 / 08 / 2 \\
021\end{array}$ & $\begin{array}{r}\mathrm{Rp} \\
689.074,29\end{array}$ & $\begin{array}{c}\mathrm{Rp} \\
589,729.988\end{array}$ & $\begin{array}{l}14.42 \\
\%\end{array}$ \\
\hline $\begin{array}{l}24 / 08 / 2 \\
021\end{array}$ & $\begin{array}{r}\mathrm{Rp} \\
696.947,92\end{array}$ & $\begin{array}{c}\mathrm{Rp} \\
613,432.104\end{array}$ & $\begin{array}{l}11.98 \\
\%\end{array}$ \\
\hline $\begin{array}{l}25 / 08 / 2 \\
021\end{array}$ & $\begin{array}{r}\mathrm{Rp} \\
685.426,79\end{array}$ & $\begin{array}{r}\mathrm{Rp} \\
591,596.102\end{array}$ & $\begin{array}{l}13.69 \\
\%\end{array}$ \\
\hline $\begin{array}{l}26 / 08 / 2 \\
021\end{array}$ & $\begin{array}{r}\mathrm{Rp} \\
687.434,13\end{array}$ & $\begin{array}{r}\mathrm{Rp} \\
575,937.022\end{array}$ & $\begin{array}{l}16.22 \\
\%\end{array}$ \\
\hline $\begin{array}{l}01 / 09 / 2 \\
021\end{array}$ & $\begin{array}{r}\mathrm{Rp} \\
685.610,96\end{array}$ & $\begin{array}{c}\mathrm{Rp} \\
610,228.510\end{array}$ & $\begin{array}{l}10.99 \\
\%\end{array}$ \\
\hline $\begin{array}{l}02 / 09 / 2 \\
021\end{array}$ & $\begin{array}{r}\mathrm{Rp} \\
686.586,44\end{array}$ & $\begin{array}{c}\mathrm{Rp} \\
603,841.771\end{array}$ & $\begin{array}{l}12.05 \\
\%\end{array}$ \\
\hline $\begin{array}{l}03 / 09 / 2 \\
021\end{array}$ & $\begin{array}{r}\mathrm{Rp} \\
685.598,10\end{array}$ & $\begin{array}{c}\mathrm{Rp} \\
595,679.698\end{array}$ & $\begin{array}{l}13.12 \\
\%\end{array}$ \\
\hline $\begin{array}{l}04 / 09 / 2 \\
021\end{array}$ & $\begin{array}{r}\mathrm{Rp} \\
683.677,41\end{array}$ & $\begin{array}{c}\mathrm{Rp} \\
581,311.976\end{array}$ & $\begin{array}{l}14.97 \\
\%\end{array}$ \\
\hline $\begin{array}{l}05 / 09 / 2 \\
021\end{array}$ & $\begin{array}{r}\mathrm{Rp} \\
683.677,41\end{array}$ & $\begin{array}{c}\mathrm{Rp} \\
595,284.703\end{array}$ & $\begin{array}{l}12.93 \\
\%\end{array}$ \\
\hline $\begin{array}{l}06 / 09 / 2 \\
021\end{array}$ & $\begin{array}{r}\mathrm{Rp} \\
683.449,08\end{array}$ & $\begin{array}{c}\mathrm{Rp} \\
594,950.870\end{array}$ & $\begin{array}{l}12.95 \\
\%\end{array}$ \\
\hline $\begin{array}{l}22 / 09 / 2 \\
021\end{array}$ & $\begin{array}{r}\mathrm{Rp} \\
671.597,84\end{array}$ & $\begin{array}{c}\mathrm{Rp} \\
603,265.017\end{array}$ & $\begin{array}{l}10.17 \\
\%\end{array}$ \\
\hline $\begin{array}{l}\text { 23/09/2 } \\
021\end{array}$ & $\begin{array}{r}\mathrm{Rp} \\
677.855,40\end{array}$ & $\begin{array}{c}\mathrm{Rp} \\
584,226.350\end{array}$ & $\begin{array}{l}13.81 \\
\%\end{array}$ \\
\hline $\begin{array}{l}24 / 09 / 2 \\
021\end{array}$ & $\begin{array}{r}\mathrm{Rp} \\
677.816,53\end{array}$ & $\begin{array}{c}\mathrm{Rp} \\
603,191.608\end{array}$ & $\begin{array}{l}11.01 \\
\%\end{array}$ \\
\hline $\begin{array}{l}25 / 09 / 2 \\
021\end{array}$ & $\begin{array}{r}\mathrm{Rp} \\
678.848,05\end{array}$ & $\begin{array}{c}\mathrm{Rp} \\
589,090.324\end{array}$ & $\begin{array}{l}13.22 \\
\%\end{array}$ \\
\hline
\end{tabular}


Gold Pricesforecasting Using Triple Exponential Method

DOI: $10.54443 /$ ijebas.v1i2.79

\begin{tabular}{|c|c|c|c|}
\hline $26 / 09 / 2$ & $\mathrm{Rp}$ & $\mathrm{Rp}$ & 12.22 \\
021 & $678.848,05$ & $595,888.565$ & $\%$ \\
\hline \multicolumn{3}{|c|}{ MAPE Average } & $\begin{array}{c}12,49 \\
\%\end{array}$ \\
\hline
\end{tabular}

The MAPE value for each date is determined from the table above, and the average MAPE value of 61 gold price data is 12.49 percent. So, based on the table above, the average error rate (MAPE) is low, and if the error rate is low, the accuracy level is high.

\section{CONCLUSION}

Forecasting on the system with the Triple Exponential Smoothing technique yields an accuracy rate of 86.93 percent and a MAPE value of 12.49 percent. The alpha and beta values are computed between 0.10 and 0.99 , yielding an alpha of 0.15 and a beta of 0.85 . This system is expected to generate output in the form of gold price data per gram in the future, making it one of the features of decision assistance for investors before investing in gold and limiting losses.

\section{ACKNOWLEDGEMENT}

Thank you to the Malikussaleh University Research and Community Service Institute which has funded this research through the PNBP Fund Source for the 2021 Budget, based on the Decree Number 530/UN45/KPT/2021 dated 12 July 2021 and the Agreement/Contract Number 114/PPK-2/SPK-JL/2021.

\section{REFERENCE}

Alameer, Z., Elaziz, M. A., Ewees, A. A., Ye, H., \& Jianhua, Z. (2019). Forecasting gold price fluctuations using improved multilayer perceptron neural network and whale optimization algorithm. Resources Policy, 61, 250-260. https://doi.org/10.1016/J.RESOURPOL.2019.02.014

ALTAN, A., \& KARASU, S. (2019). THE EFFECT OF KERNEL VALUES IN SUPPORT VECTOR MACHINE TO FORECASTING PERFORMANCE OF FINANCIAL TIME SERIES. The Journal of Cognitive Systems, 4(1), 17-21. https://dergipark.org.tr/en/pub/jcs/570863

Ayele, A. W., Gabreyohannes, E., \& Edmealem, H. (2021). Generalized Autoregressive Conditional Heteroskedastic Model to Examine Silver Price Volatility and Its Macroeconomic Determinant in Ethiopia Market. Journal of Probability and Statistics, 2021. https://doi.org/10.1155/2021/5095181

Barrow, D., Kourentzes, N., Sandberg, R., \& Niklewski, J. (2021). Automatic robust estimation for exponential smoothing: Perspectives from statistics and machine learning. Expert Systems with Applications, 160, 113637. https://doi.org/10.1016/J.ESWA.2021.113637

Chen, J., Zhu, X., \& Zhong, M. (2019). Nonlinear effects of financial factors on fluctuations in nonferrous metals prices: A Markov-switching VAR analysis. Resources Policy, 61, 489-500. https://doi.org/10.1016/J.RESOURPOL.2018.04.015

de Oliveira, E. M., \& Cyrino Oliveira, F. L. (2018). Forecasting mid-long term electric energy consumption through bagging ARIMA and exponential smoothing methods. Energy, 144, 776-788. https://doi.org/10.1016/J.ENERGY.2017.12.049

Evamelia, E., \& Panjaitan, Y. (2019). ANALISIS PERANAN EMAS DAN OBLIGASI 
PEMERINTAH SEBAGAI SAFE HAVEN PERIODE 2014-2018. BALANCE: Jurnal Akuntansi, Auditing Dan Keuangan, 16(2), 212-236. https://doi.org/10.25170/BALANCE.V16I2.1624

FAUSTINA, R. S., AGOESTANTO, A., \& HENDIKAWATI, P. (2017). MODEL HYBRID ARIMA-GARCH UNTUK ESTIMASI VOLATILITAS HARGA EMAS MENGGUNAKAN SOFTWARE R. Unnes Journal of Mathematics, 6(1), 11-24. https://doi.org/10.15294/UJM.V6I1.11681

Humphreys, D. (2017). In search of a new China: mineral demand in South and Southeast Asia. Mineral Economics 2017 31:1, 31(1), 103-112. https://doi.org/10.1007/S13563017-0118-7

Istamar, ., Sarfiah, S. N., \& Rusmijati, . (2019). ANALISIS PENGARUH HARGA MINYAK DUNIA, HARGA EMAS, DAN NILAI KURS RUPIAH TERHADAP INDEKS HARGA SAHAM GABUNGAN DI BURSA EFEK INDONESIA TAHUN 1998-2018. DINAMIC: Directory Journal of Economic, 1(4), 433-442. https://doi.org/10.31002/DINAMIC.V1I4.805

Juhro, S. M., \& Iyke, B. N. (2021). Consumer confidence and consumption expenditure in Indonesia. Economic Modelling, 89 , 367-377. https://doi.org/10.1016/J.ECONMOD.2019.11.001

Majid, R. (Rumana ). (2018). Advances in Statistical Forecasting Methods: An Overview. Economic Affairs, 63(4), 295479. https://doi.org/10.30954/0424-2513.4.2018.5

Prananingtyas, P. (2018). PERLINDUNGAN HUKUM TERHADAP INVESTOR EMAS. Masalah-Masalah Hukum, 47(4), 430-444. https://doi.org/10.14710/MMH.47.4.2018.430-444

Qasim, T. B., Iqbal, G. Z., Hassan, M. U., \& Ali, H. (2021). Application of Markov Regime Switching Autoregressive Model to Gold Prices in Pakistan. Review of Economics and Development Studies, 7(3), 309-323. https://doi.org/10.47067/reads.v7i3.368

Zhang, P., \& Ci, B. (2021). Deep belief network for gold price forecasting. Resources Policy, 69, 101806. https://doi.org/10.1016/J.RESOURPOL.2021.101806 
Volume 1 No 2 (2021)

Gold Pricesforecasting Using Triple Exponential Method DOI: $10.54443 /$ ijebas.v1i2.79 